\title{
Non-linear thermodynamic laws application to soil processes
} (Review Article)

\section{Ilgiz Khabirov a,*, Fariz Mikayilov b}

\author{
a Bashkir State Agrarian University, Department of Agronomy and Soil Science, Ufa, Russia \\ b Selçuk University, Faculty of Agriculture, Department of Soil Science and Plant Nutrition, Konya, Turkey
}

\begin{abstract}
An attempt has been made to analyze the possibility to use nonequilibrium thermodynamics for the soil dynamic open systems treatment. Entropy change of such a system and the entropy coming from or going into the outer sphere. In the steady state, dynamic soil-formation processes occur within an organized structure and are characterized by stable parameters close to equilibrium. Accordingly, when examining soil, one can proceed from the conventional thermodynamic equilibrium. However, the matter of Onzager-Prigozhin general phenomenological theory applicability to soil processes is more complicated. To study soil stability it is necessary to go beyond the limits of linear thermodynamics.
\end{abstract}

Received : 15.01 .2013

Accepted : 25.03.2013

Keywords: : Thermodynamic laws, soil processes, soil stability

(C) 2013 Federation of Eurasian Soil Science Societies. All rights reserved

\section{Introduction and overview}

The soil is a permanently or periodically open thermodynamic system where physical interactions, physicochemical, chemical and biochemical reactions are taking place. Kinetic and thermodynamic methods find more and more wide application in Soil Science and allow examining the soil genesis and fertility problems from a new point (Barbashin, 1967; Volobuyev, 1985; Kanunnikova, 1986, 1987; Savich, 1986; Chernikov, 1988; Gerayzade, 1988; Mikayilov and Khabirov, 2011).

The main difficulty in applying either chemical kinetics or thermodynamics in Soil Science lies in the absence of the unlinear irreversible processes theory which could describe both ecosystem processes far from balanced status, though at present the theory is being wored at Prigozhin, 1966; Zotin, 1984; Volobuyev, 1985; Keplen, 1986. Nevertheless this theory is not widely applicated to the soil processes analysis and, evidently, the method of classic thermodynamics will be actual for a long time.

A well-known theoretician Nobel Prize holder, one of the founders of nonequilibrium thermodynamics I.R.Prigozhin admitted in his interview to the popular scientific journal Chemistry and Life: "It took me over 15 years to believe that most nature processes sources of irreversibility within understand. As for the second principle of thermodynamics I have just started feeling that I comprehend its meaning". After those words have been said in public by a prominent scientist it is no wonder that up until now soil processes have been described accoding to the closed thermodynamic systems laws.

The methods of kinetic and thermodynamic analysis have been first applied to estimate the saccharose hydrolysis by invertaze enzyme (Khabirov, 1983). Later this approach was used to consider ecology investigations, i.e. the grey forest soils and chernozems of Suburals (Khabirov, 1986). This investigation turned to be the basis for working out new nitrification inhibitors. The increase of nitrogen fertilizers

\footnotetext{
${ }^{*}$ Corresponding author.

Bashkir State Agrarian University, Department of Agronomy and Soil Science, 450001, Ufa, Russia

Tel.: + 73472785611

E-mail address: ilkhabirov@yandex.ru
}

ISSN: $2147-4249$ 
application in Bashkiria in 1985-1990 involved the problem of plant nitrogen fertilizer used in Bashkiria the kinetic and thermodynamic characteristics of hydrolysis fermentative reaction of urea had been studied in detail (Khabirov et al., 1988; Khabirov and Kuvatov, 1990; Khabirov, 1990). To our mind the suggested article is the logical continuation of the previous investigations and has theoretical importance.

The present paper attempts to analyze the possibilities to use nonequilibrium thermodynamic principles to interprete the soil systems behaviour.

It is known that the entropy change of soil as dynamically open system is expressed by the sum of entropy produced within the system, $d_{i} S$, and the entropy coming from or going outside, $d_{e} S$

$$
d S=d_{i} S+d_{e} S
$$

where $d_{i} S$ is always a positive quantity in accordance with the thermodynamics second principle. It means that if soil sample is placed into isolation cover then $d_{e} S=0$ and system entropy can only increase. In this case $d_{i} S$ is the entropy production as the result of internal chemical reactions going on within the soil. The symbol $d_{e} S$ depends on the specific situation.

Thus, from the evolutionary aspect, soil systems are far from equilibrium and soil formation is possible only in the unbalanced system. Dynamic irreversible processes occur in the soil and as a result there appears a dynamic order with dynamic structures formation and their regulated in time behavior is possible only far from balance (Savich, 1986).

If linear equilibrium thermodynamics is justified only near balance is it justifiable to use it for soil dynamic state/processes treatment? In this case, evidently, it is necessary to consider soil as a steady-state formation, i.e. as soil moment. It means that at a definite moment stable state soil can be characterized as close to equilibrium (Smagin, 1994, 2001, 2012). At a given moment dynamic irreversibl soil - formation processes are occurring within an organized composition which changes slowly or remains practically unchanged. The possibility of stationary but nonequilibrium soil system state follows from the equation (1).

In the stationary state thermodynamics quantities characterizing the soil are permanent but do not have equilibrium values. Systems entropy is not maximum. In the stationary state $d S=0$, i.e.

$$
d_{e} S=-d_{i} S<0
$$

In other words the entropy produced goes outside completely. Accordingly, when studying the soil one may proceed from the conventional thermodynamic equilibrium. Roughly, soil formation consists of 3 stages weathering of mountain rocks, biochemical decomposition of biological structures, biosynthesis of composing elements and their formation into an organized system. Soil formation process is mostly under thermodynamic control. For example on a molecular level the system tends to the least chemical potential state. However, in the soil we find a complicated composite of thermodynamic and kinetic phenomena. The difference between thermodynamics and kinetics is illustrated by the course of a chemical reaction. The reaction is possible in principle if it is only followed by a drop in free energy, $\Delta G<0$. It is a necessary, yet insufficient condition for reaction course. If the initial and final states are divided by a high activation barrier then according to Arrhenius law

$$
k=A \exp -E_{a} / R T
$$

Where $A$-is a for-exponential multiplier, $R$-indicates a gas constant, $T$-denotes absolute temperature and can be infinitesimal. Thus one can consider nonequilibrium state protected by a high barrier, as stable. Consequently it is possible to use nonequilibrium thermodynamics to treat soil dynamic processes. To verify this assumption the following example is investigated. It is assumed that in the soil on the borderline dividing three phases (solid, liquid and gaseous) there exist tow flows namely heat flow (heat energy) and diffusion mass flow $\left(\mathrm{O}_{2}\right.$ absorption and $\mathrm{CO}_{2}$ release). $J_{1}$ - denotes heat flow and $J_{2}$-indicates mass flow.

These flows correspond to two generalized forces-ttemperature difference (between soil and air) and concentration difference $X_{1}$ and $X_{2} \quad\left(\mathrm{O}_{2}\right.$ and $\mathrm{CO}_{2}$ partial pressure). Generalized flows $J_{1}$ depend on the generalized forces $\left(X_{j}\right)$, and vice versa, biochemical reaction rate depends on affinity between ferment and substratum, acidity, substratum concentration, temperature, heat energy flow depends on temperature difference. In general this dependence is expressed by the following ratio (Volkenstein, 1981) 


$$
J_{i}=\sum_{j=1}^{n} L_{i j} X_{j} \quad j=1,2, \ldots, n
$$

where $L_{i j}$ - denotes phenomenological coefficients.

Since both heat and mass flows depend on both temperature difference and concentration difference - they are conjugate:

$$
\begin{aligned}
& J_{1}=L_{11} X_{1}+L_{12} X_{2} \\
& J_{2}=L_{21} X_{1}+L_{22} X_{2}
\end{aligned}
$$

Accoding to Onzager's theorem when close to balance phenomenological coefficient $\mathbf{L}_{\mathbf{i j}}$ form a symmetric matrix, i.e.

$$
\mathbf{L}_{\mathrm{ij}}=\mathbf{L}_{\mathbf{j i}}
$$

This proposition follows directly from microscopic reversibility. Invers ratios can the expressed by

$$
X_{i}=\sum_{j=1}^{n} R_{i j} I_{j}
$$

where $\mathbf{R}_{\mathrm{ij}}$ - coefficient also forms a symmetric matrix.

Dissipation function being $\sigma \geq 0$, phenomenological coefficient $\mathbf{L}_{\mathrm{ij}}$ (and accordingly $\mathbf{R}_{\mathrm{ij}}$ ) are subjected to definite conditions.

When examining without affinity limitations the case of two forces and two flows (5 and 6), then according to the formula

$$
\sigma=\sum_{j=1}^{n} I_{j} X_{j}
$$

where $\sigma$ - denotes dissipation function, i.e. entropy production rate in a volume unit, we have

$$
\sigma=L_{11} X_{1}^{2}+L_{12}+L_{21} X_{1} X_{2}+L_{22} X_{2}^{2} \geq 0
$$

Dissipation function $\sigma-$ is positive under any differing from zero variable values $X_{1}, X_{2}$ and it turns into zero only if $X_{1}=X_{2}=0$. Therefore,

$$
\begin{gathered}
L_{11}>0, L_{22}>0 \\
L_{12}+L_{21}{ }^{2}<4 L_{11} \cdot L_{22}
\end{gathered}
$$

In consequence of $L_{12}=L_{21}$ coefficient symmetry,

$$
L_{12}+L_{21}^{2}<4 L_{11} \cdot L_{22} \Leftrightarrow L_{12}^{2}<L_{11} \cdot L_{22}
$$

Any value can be nondiagonal coefficient value. In general case:

$$
\mathbf{L}_{\mathrm{ij}}>0, \mathbf{L}_{\mathrm{ii}} \mathbf{L}_{\mathrm{j} \mathbf{j}}>\mathbf{L}_{\mathrm{ij}}
$$

It should be underlined that the conditions $\sigma=0$ refer to the sum $\sum_{i} \mathbf{X}_{\mathbf{i}} \mathbf{J}_{\mathbf{i}}$ in general.

Separate terms of this sum may be negative. It meens that a separate separate flow $\mathbf{J}_{\mathbf{i}}$ is impossible as $\mathbf{X}_{\mathbf{i}} \mathbf{J}_{\mathbf{i}}<0$. In other words such a flow would be contradictory to the second principle. However, owing to the conjugation with other flows that correspond to positive values $\mathbf{X}_{\mathbf{i}} \mathbf{J}_{\mathbf{i}}>0$, the flow impossible in the closed system is possible in the open system. The following condition must be carried out when

$$
\sum_{i} \mathbf{X}_{\mathbf{i}} \mathbf{J}_{\mathbf{i}}>\left|\mathbf{X}_{\mathbf{i}} \mathbf{J}_{\mathbf{i}}\right|
$$

For example, in the process of nitrogen fixation, molecular nitrogen transportation through micro-organisms cells membranes means that $\mathrm{N}_{2}$ ion diffusion flux is conjugated with fermentative reaction of ATPh breaking up, and that amino acids policondensation is conjugated again with ATPh hydrolysis. Entropy deficiency in one process exceeds its surplus production in another one. Open system entropy production ensures 
processes which are impossible in isolated system. This proposition is important for soil processes understanding.

If the system is close to equilibrium (stationary climax soil state) and Onzager's theorem is realized, i.e. $\mathbf{L}_{12}=\mathbf{L}_{21}$, then we get

$$
\frac{\partial \sigma}{\partial X_{m}}=2 \mathbf{L}_{12} \mathbf{X}_{\mathbf{T}}+\mathbf{L}_{22} \mathbf{X}_{\mathrm{m}}=2 \mathbf{J}_{\mathrm{m}}=0
$$

The second derivative of this equation is

$$
\frac{\partial^{2} \sigma}{\partial X_{m}^{2}}=2 \mathbf{L}_{22}>0
$$

Therefore in stationary state close to equilibrium, entropy production is minimum. This is Prigozhin's theorem. It is in direct connection with Onzager's theorem and is valid only within the limits of linear thermodynamics applicability, i.e. when $\mathbf{L}_{\mathbf{i j}}$ to $\mathbf{X}_{\mathbf{i}}$ coefficients are irreplaceable.

The first equation (4) describes different kinetic processes conjugation as the result of nondiagonal coefficients $\mathbf{L}_{\mathrm{ij}} i \neq j$ difference from zero and the second (16) one is a mathematical expression of Prigozhin's theorem on entropy production minimum in stationary state. Undoubtedly that conjugate processes are realized in the soil as in the open system. That is why Onzager - Prigozin's General phenomenological theory applicability to soil system in more complicated as entropy production $\sigma$ is minimal only in those stationary states of biological system which are close to equilibrium. These systems are described by linear ratios (4). But to investigate soil systems and their dynamic order it is essential to go beyond the limits of linear thermodynamics.

In the process of soil evolution there appears a dynamic order, new types of substance organization in time and space, characteristic of only open, far from equilibrium system called dissipative systems. This state is supported by the entropy release into the outer sphere exceeds entropy production within the system.

It is the most probable for soil system:

1. Entropy release increase with the simultaneous growth of free energy owing to humus and clayey minerals accumulation.

2. Free energy growth with the simultaneous entropy decrease within the system that can be only the result of additional energy flow into the system.

3. Close to equilibrium system behavior as being described within the limits of linear thermodynamics. We are dealing here with equilibrium structures modified as a result of limitations blocking equilibrium achievement (for example, complete equilibrium in soil solution); pH level, soil buffer, $\boldsymbol{C}_{\mathrm{ga}}: \boldsymbol{C}_{\mathrm{fa}}$ et cetera. Under such conditions dynamic order is impossible - stationary states are asymptomatically stable. In such cases it is possible to use Prigozin's theory.

4. Dynamic order occurs in dissipative system. A criterion of possibility of dynamic order appearance in dissipative system is not is not to fulfill stability conditions. Let's examine again some of the open system thermodynamics ratios. Dissipation function equals

$$
\sigma=\frac{d_{i} S}{d t}=\sum_{j} \mathbf{J}_{\mathbf{j}} \mathbf{X}_{\mathbf{j}} \geq 0
$$

Its change in time is written as follows

$$
\frac{d \sigma}{d t}=\frac{d_{\imath} \sigma}{d t}+\frac{d_{x} \sigma}{d t}+\sum_{j} \mathrm{X}_{j} \frac{d J_{j}}{d t}+\sum_{j} J_{j} \frac{d \mathrm{X}_{j}}{d t}
$$

When under constant border conditions we have

$$
\frac{d X \sigma}{d t} \leq 0
$$

Stationary state stability condition under examination follows from here

$$
\sum_{j} \delta J_{j} \delta X_{j} \geq 0
$$

Where $\delta J_{j}, \delta X_{j}$ denote generalized flows and forces deviations from their stationary values. 
When close to equilibrium this condition is always fulfilled. Applied to chemical processes it looks like

$$
\sum_{j} \delta V_{j} \delta A_{j}
$$

If the condition (21) is not fulfilled, stationary state is unstable and fluctuation intensification leading to dynamic order origin is possible. "Order through fluctuations" is obviously possible only in such an open system as soil for its behaviour is considerably unlinear. In soil we deal with several types of phenomena showing directly that the corresponding processes are unlinear.

Firstly, these are any threshold processes of switching soil systems from one condition into another.

Secondly, humus, Nitrogen-organic and other substances systems behaviour at all levels of soil formation is subject to regulation and control from the part of soil-formation factors being defined in particular by reverse connections which are missing in linear system.

Thirdly, these are periodic oscillatory phenomena. At all levels of soil system organization from molecular to typical, there occur non fading oscillations of characteristic parameters - fermentative activity, metabolite concentration, micro organism numbers, nutrition elements contents, et cetera.

Fourthly, soil system, beginning with elementary soil formation processes and finishing with the biosphere as a whole, is developing irreversibly, is evolving. Development always means new structures origin, new information "soil memory, soil-moment" creation, i.e. the processes are considerably nonlinear.

Apparently, it is necessary to examine multiple stable and unstable stationary states of soil systems and transition between them. As a matter of fact thermodynamic comes to an end here - it is not sufficient to describe nonlinear systems on its basis. Thermodynamics allows only to formulate stability criteria. To further investigate self organizing systems it is necessary to use physico-mathematical modeling, to build dynamic models.

\section{References}

Barbashin, E.A., 1967. Intraduction into stability theory. M.Nauka, pp.223.

Chernikov, V.A., 1988. Soil humus state evalution from thermodynamics and kinetics point. The Timiryazev Agricultural Academy proceedings, 5, 49-58.

Gerayzade, A.P., 1988. Energy transformation in the system soil-plant-atmosphere. The author's dissertation abstract. M. Moscow State University, p.31

Kanunnikova, N.A., 1986. Thermodynamic potential of soil reactions and soil buffer properties. Science and Technology Review. Soil and Agrochemistry M. VINITI, pp. 87-184.

Kanunnikova, N.A., 1987. Soil nitrogen potential. Soil study. 3, 58-67.

Keplen, S.R., 1986. Essing E.: Irreversible processes Bioenergetics and linear thermodynamics. M.: Mir, pp.382.

Khabirov, I.K., 1983. Kinetic and thermodynamic characteristics of the Saccharase reaction in Soils. Soil Science 10, 141146.

Khabirov, I.K., 1986. Kinetics and temperature dependence of nitric nitrogen accumulation in Sub-Urals soils. Soil Science 5, 129-135.

Khabirov, I.K., Prostykova Z.G, Kaspransky, N.N., 1988. Potentially mineralizable nitrogen compounds in cis Uralian Soils. Soviet Soil Science 20(3), 91-99.

Khabirov, I.K., Kuvatov, Yu. G., 1990. Kinetics and thermodynamics of the hydrolysis reaction of ${ }^{14} \mathrm{C}$-Labelled Carbamide in the Pre-Urals Soils. Soil Science, 8, 83-94.

Khabirov, I.K., 1990. Application of kinetic methods for nitrogen transformation processes evalution in soils. Soil Science, 12, 112-119.

Mikayilov, F.D., Khabirov, I.K. 2011. Some questions of modeling of enzymatic processes in soil. The III International scientific - practical conference 'Soil as connecting part for natural and anthropogenic - transformed ecosystems co-functioning', devoted to 80-year anniversary of Department of Pedology and Land Resources Estimation of Irkutsk State University. August, 15-22, 2011, Irkutsk, Russia. Scientific publications, pp. 166 - 171.

Prigozhin, J., Defe,G., 1966. Chemical thermodynamics, Novosibirsk: Nauka, pp.509.

Savich, V.I., 1986. Thermodynamics of ion combination transformation in soil. Science and technology results. Soil Science and Agrochemistry, M.: VINITI, 6, 7-86.

Smagin, A.V., 1994. The Theory of soil stability. Soil Science, 12, 26-34.

Smagin, A.V.,etal. 2001. Modelirovanie dinamiki organicheskogo veshestva pochv1, 300dpi,120p

Smagin, A.V., 2012. Theory and Practice of soil engineering. - M.: Moscow State University Press, 544 p.

Volkenstein, M.V., 1981. Biopyysics. M.: Nauka, pp. 575

Volobuyev, V.R., 1985. On biological component of soil formation energetics. Soil Study 9, 5-8

Zotin, A.J., 1984. Biological processes thermodynamics and regulation. M.: Nauka, pp. 334. 